\title{
Machines-outils à structure parallèle et usinage à grande vitesse
}

\author{
Myriam Terrier, Arnaud Dugas et Jean-Yves Hascoëta \\ Institut de Recherche en Communications et Cybernétique de Nantes (IRCCyN), UMR CNRS 6597, 1 rue de la Noë, BP 92101, \\ 44321 Nantes Cedex 03, France
}

Reçu le 14 février 2005, accepté le 13 avril 2005

\begin{abstract}
Résumé - Depuis la présentation publique de la première machine-outil à structure parallèle à Chicago en 1994, peu d'études ont été rendues publiques quant à leurs potentiels en usinage, de même que peu de machines réalisent actuellement des opérations d'usinage dans l'industrie. L'objectif de cette étude est d'analyser les potentialités de ces machines en usinage, et en particulier en usinage à grande vitesse de formes complexes. Pour cela, une méthode de mesure, précédemment testée et validée sur des machines sérielles et basée sur l'exploitation des sorties des codeurs de la machine, a été utilisée. Des essais ont été réalisés sur cinq machines sérielles et quatre machines parallèles, ce qui a permis de dresser un premier bilan. À l'heure actuelle, il a été montré que les machines parallèles peuvent produire des résultats intéressants en terme de temps d'usinage et de précision, et que les paramètres $\mathrm{CN}$ et $\mathrm{FAO}$ ont une influence non négligeable sur la «qualité » de la pièce finale. Il devient donc impératif de développer des procédures génériques afin de tester la réelle aptitude de chaque machine. Le simulateur qui a été développé précédemment au sein de notre équipe pour les machines sérielles est complété pour l'analyse des machines à structure parallèle.
\end{abstract}

Mots clés : Machine-outil à structure parallèle / usinage à grande vitesse / qualification de machines/ formes complexes

\begin{abstract}
PKM machine and High Speed Machining. Since the first presentation of parallel kinematics machines tools (PKMs) in Chicago in 1994, very few papers have dealt with their behaviour in milling. Moreover, very few are used nowadays in industry. The objective of this study is to show the potentialities of PKMs in milling, and especially in high-speed milling of free form surfaces. In order to do so, a method of measurement - previously tested and validated on serial machines (SKMs) - that needs the encoder data of the machine, has been used. Experiments on five SKMs and four PKMs have been conducted, which allowed to make a first comparison. Though, it is important to note that the goal of this study is not to judge the PKMs but to study their potentialities in milling in order to develop their use in industry. So far, it has been shown that PKMs can provide interesting results in terms of time and precision, and that the NC and CAM parameters could have an influence, which cannot be neglected, on the final part. This is the reason why it is important to define generic procedures in order to test the real aptitude of the machines. Hence, the simulator that has been developed previously in our team is extended for PKMs.
\end{abstract}

Key words: Parallel kinematics machine / high-speed milling / machine qualification / free form surface

\section{Introduction}

Depuis 1994, date de la présentation publique de la première machine-outil à structure parallèle, de nombreux prototypes ont été développés. Cependant très peu d'entre eux sont actuellement utilisés dans l'industrie et lorsque cela est le cas, ces machines réalisent principalement des opérations de perçage, lamage, taraudage mais

\footnotetext{
a Auteur pour correspondance :

Jean-Yves. Hascoet@irccyn.ec-nantes.fr
}

pas ou peu d'usinage de formes. Pourtant, l'intérêt majeur de ces machines réside dans leurs caractéristiques dynamiques qui pourraient s'avérer très intéressantes dans le développement de l'usinage à grande vitesse de formes complexes.

L'objectif principal de ce travail est d'étudier le potentiel des structures parallèles dans le domaine de l'usinage. Pour ce faire, une nouvelle méthode de mesure qui s'appuie sur des enregistrements des sorties des codeurs a été utilisée pour qualifier les machines-outils, cette méthode 
a été précédemment testée et validée sur des machinesoutils sérielles [1]. Des essais ont été réalisés sur quatre machines à structure parallèle ce qui a permis dans un premier temps de tester la méthode de mesure sur ces nouvelles structures, et dans un second temps de comparer le comportement de ces machines aux références actuelles qui sont les machines sérielles. Il est important de préciser ici que le but de cette étude n'est pas de juger les machines-outils parallèles, mais d'évaluer leurs potentialités en usinage afin de les développer pour l'industrie. Ceci explique le choix de garder l'anonymat de l'ensemble des machines testées, et ce quelle que soit leur structure. De plus, tous les résultats fournis ne sont que le reflet des machines à l'instant où nous avons effectué les tests.

Dans un premier temps, un rapide tour d'horizon des mécanismes parallèles est effectué, puis le protocole expérimental est présenté afin de conclure avec l'analyse des premiers essais et en particulier les résultats obtenus en terme de temps d'usinage et de précision.

\section{Les mécanismes parallèles}

Merlet définit un manipulateur parallèle comme un mécanisme en chaîne cinématique fermée, constitué d'un organe terminal à $n$ degrés de liberté et d'une base fixe, reliés entre eux par au moins deux chaînes cinématiques indépendantes [2]. Si le nombre de chaînes cinématiques indépendantes est égal au nombre de degrés de liberté alors le manipulateur est dit pleinement parallèle, s'il est strictement inférieur il est dit hybride. Ainsi, les manipulateurs parallèles se différencient des manipulateurs sériels dont l'architecture consiste en un empilement de segments motorisés.

\subsection{Applications}

De part la littérature, les structures parallèles sont particulièrement intéressantes puisqu'elles offrent de bonnes performances dynamiques (c'est-à-dire une bonne précision, une grande rigidité et de grandes vitesses et accélérations). Ainsi, elles sont utilisées dans des domaines variés tels que l'assemblage, la mécanique, la médecine... [3]. Selon la tâche à réaliser, une configuration de structure parallèle sera plus ou moins adaptée (exemple : Delta pour des opérations d'assemblage [4], Plate-Forme de Gough-Stewart pour les simulateurs de vol...). Dans le domaine de l'usinage, le développement de machines-outils à structure parallèle est particulièrement intéressant pour l'usinage à grande vitesse puisque l'outil est supporté par tous les axes. Ainsi, la masse supportée par chaque axe est moins importante que pour une machine sérielle.

Depuis 1994, de nombreux prototypes basés sur différentes configurations de structure parallèle (Hexapode comme la $6 \mathrm{X}$ de Mikromat, Delta linéaire comme l'UraneSX de Renault Automation, Hybride comme la
Tricept de SMT Tricept...) ont été développés à travers le monde. Différents points de vues et études, principalement basés sur des critères théoriques (espace de travail, rigidité...), ont permis de faire ressortir les avantages et les faiblesses de ces structures [5-7]... Le principal inconvénient qui freine l'utilisation des structures parallèles en usinage est lié au fait que tous les avantages listés précédemment ne sont que potentiels. Un robot parallèle n'aura en pratique de très bonnes performances que si tous ces composants présentent un haut niveau de compétence [3]. Ainsi, parmi le lot de prototypes de machines-outils à structure parallèle, certains sont capables d'usiner des pièces dans des matériaux tels que l'aluminium ou l'acier tandis que d'autres n'en sont pas encore à ce stade. De même, très peu de ces machines sont actuellement utilisées dans l'industrie, la plus commercialisée est celle développée par SMT Tricept (utilisation dans les domaines automobile et aéronautique) [8].

Une enquête [9] a permis aux auteurs de collecter des informations sur 46 machines parallèles; cependant, très peu de fabricants donnent la répétabilité et la précision de leurs machines, et lorsque cela est le cas il s'agit souvent de données théoriques. Ce silence ne fait qu'accroître la difficulté à estimer le potentiel de celles-ci. Une étude réalisée au Royaume-Uni, dans le cadre du projet européen RAMOULDIE, a récemment comparé les performances de l'hexapode Variax avec une machine sérielle 3 -axes et une machine sérielle 5-axes [10]. Les auteurs ont montré la difficulté à comparer des machines-outils dont les structures et les commandes numériques sont différentes. La conclusion de cette étude est que pour la pièce test utilisée et pour les critères choisis, l'hexapode a un niveau de précision similaire aux deux autres machines mais pas meilleur, ce qui constitue déjà un grand pas en avant. Cependant, d'autres études sur d'autres machines parallèles sont nécessaires afin de généraliser, si possible, le comportement de ces machines ou au moins afin de montrer l'éventuel intérêt de ces structures en usinage, c'est-à-dire vérifier si le niveau de précision obtenu est équivalent à celui des machines sérielles pour une dynamique plus élevée.

\subsection{Notre domaine d'investigation}

L'objectif principal de cette étude est de qualifier les machines parallèles par rapport aux machines sérielles dans le domaine de l'usinage. En d'autres termes, il s'agit de répondre aux questions suivantes : Est-ce que les machines parallèles sont plus efficaces (en terme de précision, productivité...) que les machines sérielles? Est-ce le cas pour tous les types d'opérations (formes complexes, perçage...), pour toutes les configurations d'usinage (2-axes à 5-axes), pour tous les types de matériaux, ...? Existe-t-il une adéquation entre la structure parallèle et la pièce à usiner? En effet, on considère que les structures hexapodes sont particulièrement adaptées pour les simulateurs de vol, en est-il de même pour l'usinage ou existe-t-il une structure plus adaptée pour un type particulier d'opération (perçage, fraisage...)? 


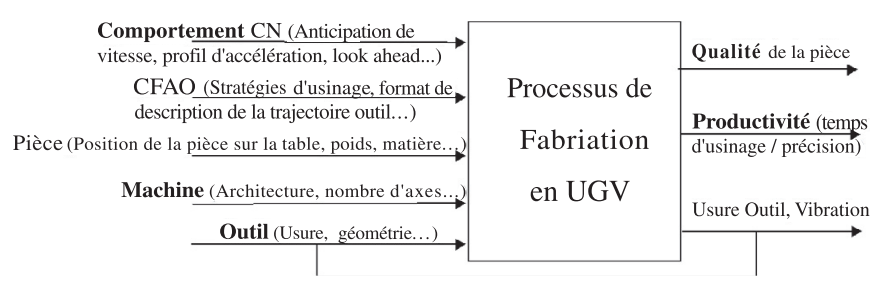

Fig. 1. Processus de fabrication en UGV.

Pour ce faire, le processus d'usinage est considéré dans son ensemble (cf. Fig. 1). Tout ceci permettra à terme d'optimiser le processus de fabrication sur les machinesoutils parallèles et sérielles.

\section{Protocole expérimental}

\subsection{Méthode de qualification de machine}

Au cours d'une précédente étude, une nouvelle méthode pour qualifier les machines (i.e. pour vérifier leurs aptitudes à réaliser une pièce dans une tolérance) a été développée $[11,12]$. Le principe consiste à récupérer pour chaque axe la position réelle de l'outil en collectant les données à la sortie des codeurs pendant un usinage. Ceci permet d'obtenir la trajectoire réellement suivie par l'outil. Comme le montre la figure 2, la trajectoire ainsi obtenue peut être comparée au fichier ISO en calculant l'erreur de contour [1].

L'intérêt majeur de cette méthode est qu'elle permet de qualifier la machine, et en particulier l'influence de sa structure, dans des conditions réelles d'usinage et sur des formes complexes. Cependant, celle-ci est à utiliser en complément du contrôle par MMT puisqu'elle intervient en amont de l'outil (la mesure par MMT intervenant en aval). L'étude des machines parallèles a été basée sur cette méthode qui a été précédemment testée et validée sur trois machines sérielles [1].

\subsection{Géométrie de la pièce test}

Tous les essais ont été conduits sur la même pièce test dont la particularité est d'être composée de différentes caractéristiques géométriques (Fig. 3). Cette pièce a été conçue en collaboration avec le LURPA $[13,14]$. Elle regroupe différents types de formes complexes sollicitant la machine à des degrés divers afin de caractériser au mieux le comportement de celle-ci [11].

\subsection{Machines-outils et paramètres testés}

À l'heure actuelle, nous avons eu l'opportunité de réaliser des essais, basés sur le même fichier ISO, sur six machines sérielles et quatre machines parallèles. Par soucis de confidentialité, nous les avons rendues anonymes et notées $S K M X-y$ pour les machines sérielles et $P K M X-y$ pour les machines à architecture parallèle comme le montre le tableau 1. L'utilisation du double indice $(X-y)$ pour désigner les machines permet de mettre en évidence que la machine $(X)$ est la même mais que les paramètres $\mathrm{CN}(y)$ sont différents. Quand cela a été possible ( $S K M A, S K M B$ et $P K M H)$, une pièce a été usinée dans un alliage d'aluminium (2024). Sur $P K M G$, la pièce a été usinée dans un bloc de résine, et sur $S K M C$ - $a$ dans un bloc d'acier (40CMD8S - AFNOR). En ce qui concerne les paramètres $\mathrm{CN}$, la difficulté réside dans le fait qu'ils sont propres à celle-ci et donc différents d'une $\mathrm{CN}$ à une autre (tolérance CN, compactage des blocs...). Cependant, nous allons montrer par la suite que notre méthode permet d'optimiser la machine en faisant varier ces divers paramètres et donc de rechercher la meilleure configuration en fonction d'un critère donné (temps, précision... ).

\section{Remarques}

- La carte du microprocesseur de $S K M B$ - $b$ est plus puissante que celle de $S K M B-a$.

- La nuance entre $S K M C$ - $a$ d'une part et $S K M C$ - $b$ et $S K M C$-c d'autre part vient du fait que ce sont deux machines de même modèle mais se trouvant sur deux sites différents.

- PKM H est la seule machine pour laquelle les sorties codeurs n'ont pas été relevées.

\section{Résultats}

\subsection{Visualisation des résultats}

Comme le montre la figure 4 (en haut à droite), l'erreur de contour peut être représentée dans un graphique en fonction de l'abscisse curviligne. Ce moyen de visualisation sera utilisé pour une étude quantitative, par contre afin de comparer plus aisément le comportement de chaque machine nous allons utiliser les trois graphiques présentés ci-après. L'analyse des résultats est donnée dans les sections 4.2 et 4.3 .

\subsubsection{Représentation de l'erreur de contour}

La plage de l'erreur de contour est définie comme l'amplitude du signal, c'est-à-dire l'erreur de contour maximum moins l'erreur de contour minimum (cf. Fig. 4 en haut à droite).

La figure 4 donne la plage de l'erreur de contour pour chaque machine en fonction du profil considéré de 1 à 4 (cf. Fig. 3). Dans un premier temps, nous ne tiendrons pas compte du sens de parcours, ainsi les résultats présentés correspondent au cas où la stratégie adoptée serait du zigzag (moyenne entre l'aller et le retour). L'axe des abscisses correspond aux machines, tandis que l'axe des ordonnées correspond à la plage de l'erreur de contour exprimée sous forme de «pourcentage ». Pour cela, soit $A_{i j}$ la plage de l'erreur de contour pour la Machine $j$ et le Profil $i$, le 


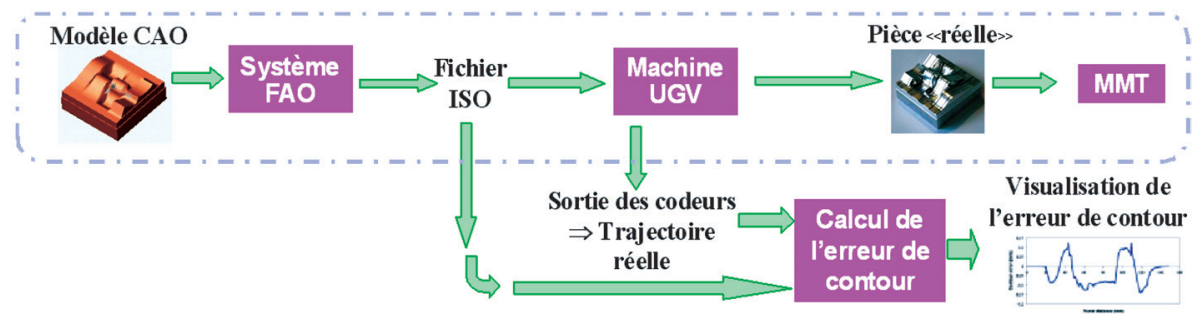

Fig. 2. Principe de la méthode de qualification de machine.

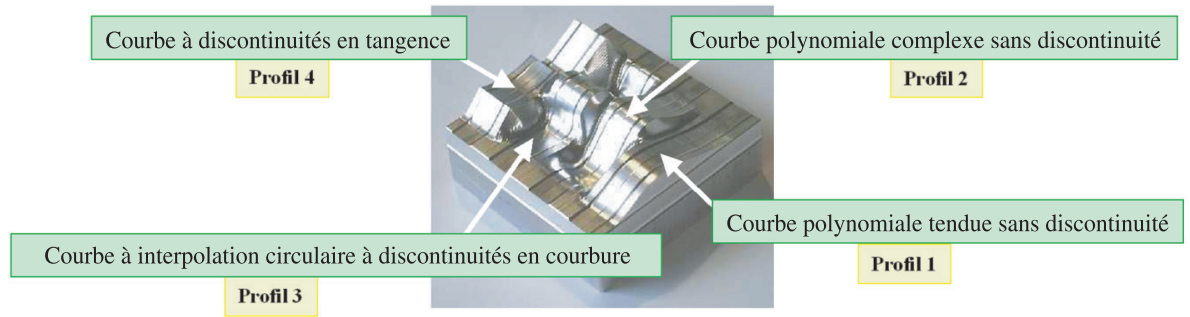

Fig. 3. Géométrie de la pièce test.

Tableau 1. Paramètres testés sur chaque machine.

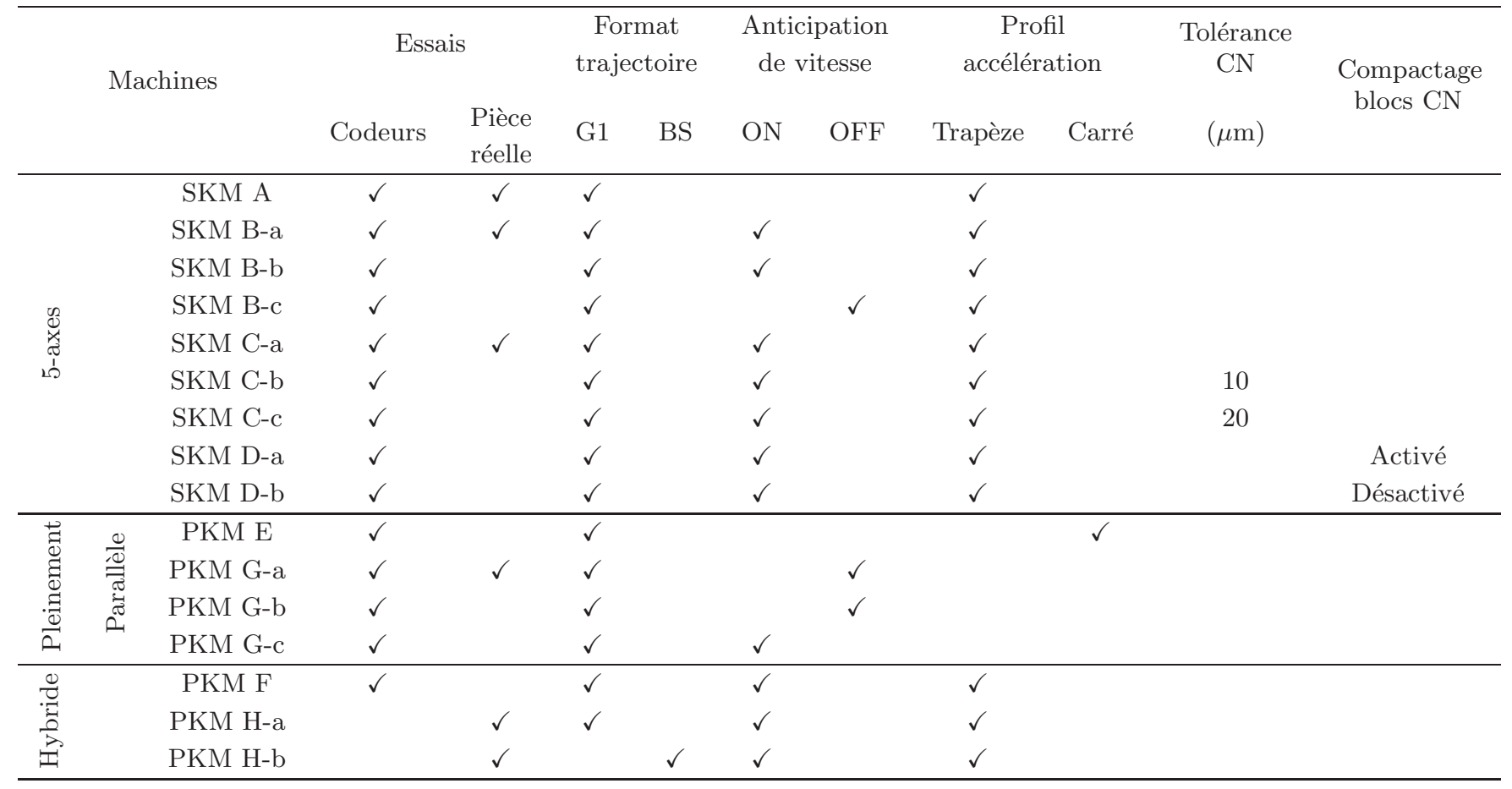

maximum $\operatorname{Max} A_{i j}$ est alors rendu égal à 100 . Ce même changement d'échelle est appliqué à tous les autres $A_{i j}$. Ainsi, plus $A_{i j}$ est proche de zéro, plus la Machine $j$ (pour le Profil $i$ ) est précise.

\subsubsection{Représentation du temps d'usinage}

La figure 5 donne le temps d'usinage - calculé à partir du relevé des codeurs - pour chaque machine en fonction du profil considéré. Comme précédemment, l'axe des abscisses correspond aux machines, tandis que l'axe des ordonnées correspond au temps d'usinage exprimé sous forme de «pourcentage » (changement d'échelle réalisé comme pour l'erreur de contour).

Ainsi, si l'on note $T_{i j}$ le temps d'usinage pour la $M a$ chine $j$ et le Profil $i$, plus $T_{i j}$ est proche de zéro, plus la Machine $j$ (pour le Profil $i$ ) est rapide.

\subsubsection{Représentation de la productivité (temps/précision)}

Un critère unique, noté $P_{i j}$, reliant le temps et la précision a également été mis en place afin de proposer 


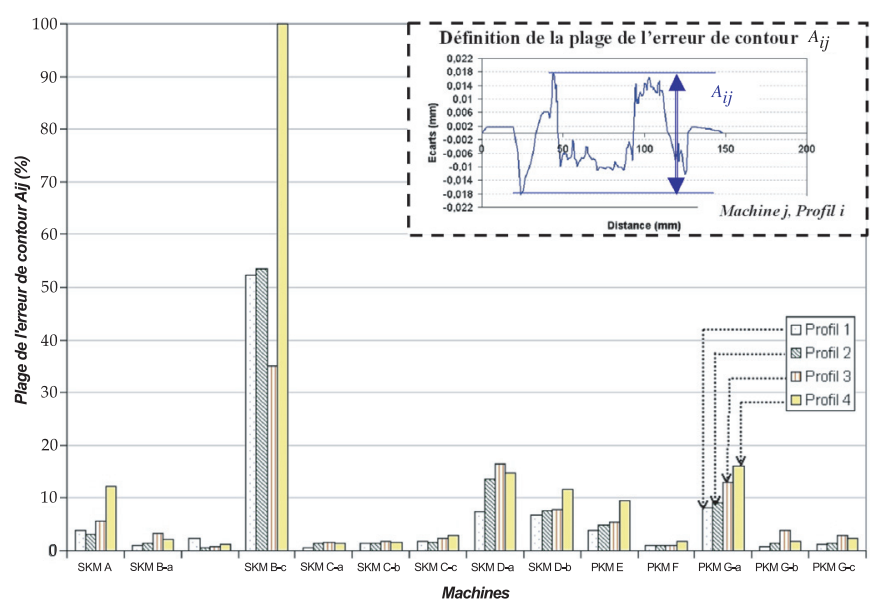

Fig. 4. Plage de l'erreur de contour par machine et par profil.

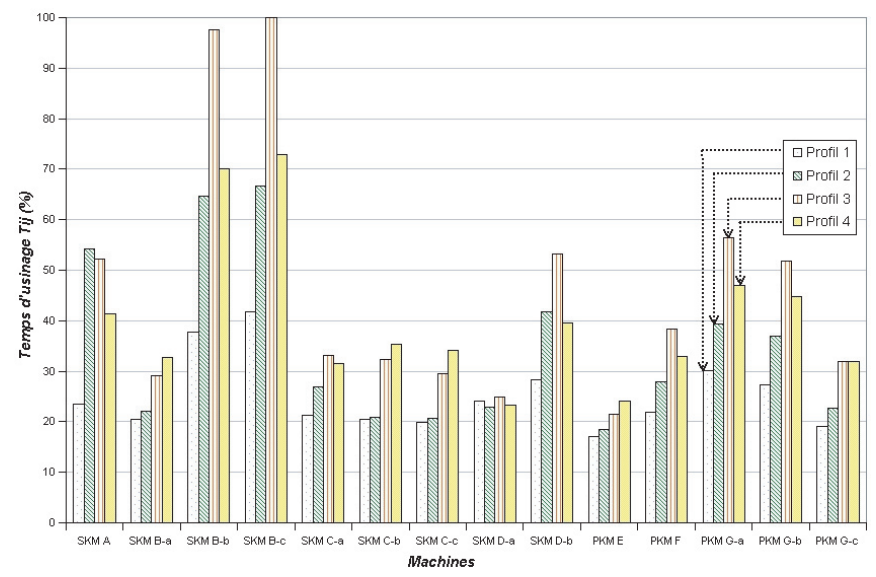

Fig. 5. Temps d'usinage par machine et par profil.

un indicateur de productivité de la machine. Pour cela, un indice de précision $C p_{I T}$ a été défini tel que :

$$
C p_{I T}=\frac{1}{E\left(\frac{\text { Plage }}{I T}\right)+1} \text { où } E(X) \text { est la partie }
$$

entière de $X$.

La plage correspond à la plage de l'erreur de contour du trajet considéré et l'IT correspond à une référence fixée au préalable (ici, nous avons fixé $I T=0,01 \mathrm{~mm}$ ), de ce fait $\left.\left.C p_{I T} \in\right] 0 ; 1\right]$. Ainsi si une machine a une plage d'erreur de contour comprise entre 0 et $0,01 \mathrm{~mm}$, l'indice de précision à $0,01 \mathrm{~mm}$ vaut 1 . Plus la machine est précise et plus $C p_{0,01}$ est grand et proche de 1 .

L'indice de précision étant défini, il est alors possible de calculer le rapport $P_{i j}$ entre le temps d'usinage et l'indice $C p_{0,01}$ tel que $P_{i j}=\frac{T_{i j}}{C p_{0,01}}$. Ainsi, plus $P_{i j}$ est proche de zéro, plus la Machine $j$ (pour le Profil $i$ ) est productive.

Ce rapport va permettre de déterminer quelle est la « meilleure » machine en tenant compte à la fois du temps d'usinage et de la précision. Les figures 6 et 9 représentent pour chaque machine : $P_{i j}$ ainsi que la valeur mini, maxi et la moyenne de $P_{i j}$ sur les quatre profils.

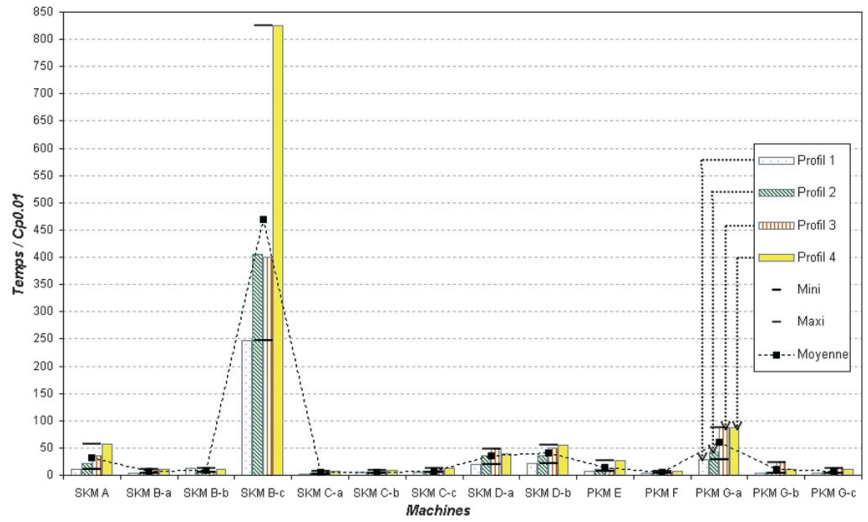

Fig. 6. $P_{i j}=\left(\right.$ Temps d'usinage $\left./ C p_{0,01}\right)$ par profil.

\subsection{Influence des paramètres FAO et $\mathrm{CN}$}

Toutes les pièces ont été usinées à partir du même fichier ISO. Il s'avère que ce fichier n'est pas suffisant pour reproduire exactement le même usinage d'une machine à l'autre. En effet, l'opérateur peut jouer sur différents paramètres au pied de la machine (potentiomètre des avances, paramètres CN...). Des précautions particulières doivent donc être prises avant tout usinage, car certains de ces paramètres peuvent influencer le résultat final. C'est le cas, par exemple, du paramètre d'anticipation de vitesse (feed forward $=f f w$ ) comme le prouve la comparaison entre $S K M B-b(f f w O N)$ et $S K M B-c(f f w O F F)$. La figure 7 donne les courbes d'écarts dans le cas du profil 3.

De la même manière, la figure 8 donne l'erreur de contour calculée à partir d'un relevé MMT (Machine à Mesurer Tridimensionnelle) des deux pièces $P K M H$ - $a$ et $P K M H$-b. Sur cette machine le paramètre testé est le format de description de la trajectoire de l'outil (linéaire ou B-Spline) qui est lié à la FAO et donc stocké dans le fichier ISO. Sur cette machine, l'utilisation du format B-Spline permet de réduire l'erreur de contour.

La méthode de mesure qui utilise les codeurs de la machine trouve ici tout son intérêt. En effet, même s'il existe une différence entre les données codeurs et la pièce réelle [1], l'avantage est qu'il n'est pas nécessaire d'usiner une pièce pour tester le comportement de la machine. Il est donc possible d'optimiser le comportement de celle-ci en faisant varier les paramètres d'usinage, et ensuite de confirmer les résultats obtenus par les codeurs en usinant une pièce et en la mesurant via une MMT. Cette méthode a été appliquée en partie sur la $P K M G$. Les résultats ont été concluants puisque la modification de certains paramètres $\mathrm{CN}$ a permis de diviser par quatre la plage de l'erreur de contour entre PKM G- $a$ et $P K M G$-c, tout en réduisant légèrement le temps d'usinage (cf. Figs. 4-6).

La figure 9 correspond à un zoom de la figure 6 qui permet de mieux visualiser, entre autre, l'influence du processeur de la carte du contrôleur ( $S K M B$ - $a$ et $S K M B$ - $b)$. L'utilisation d'un processeur plus puissant $(S K M B-b)$ a permis de gagner en précision (au détriment du temps d'usinage). 


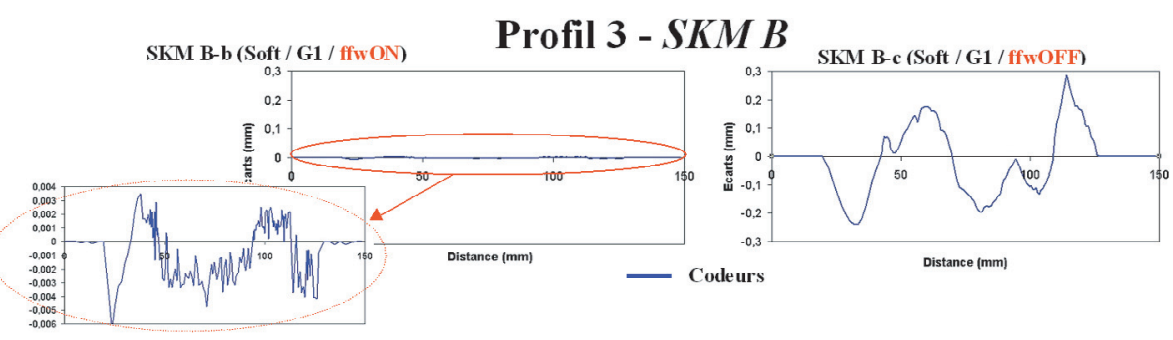

Fig. 7. Influence de l'anticipation de vitesse (SKM B).

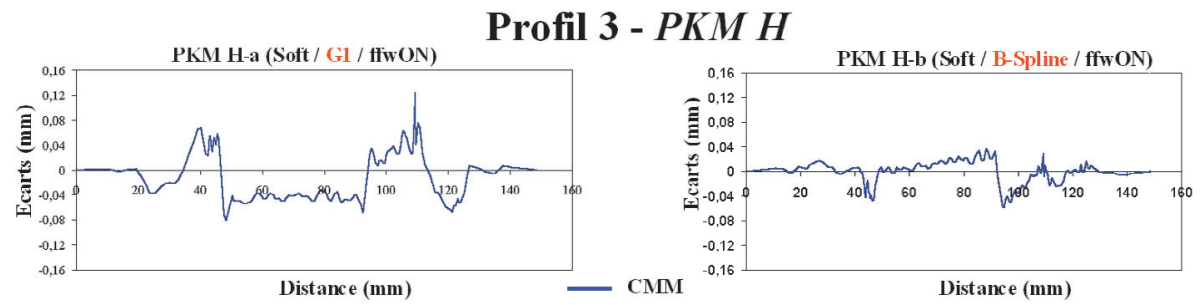

Fig. 8. Influence du format de description G1/B-Spline ( $P K M H)$.

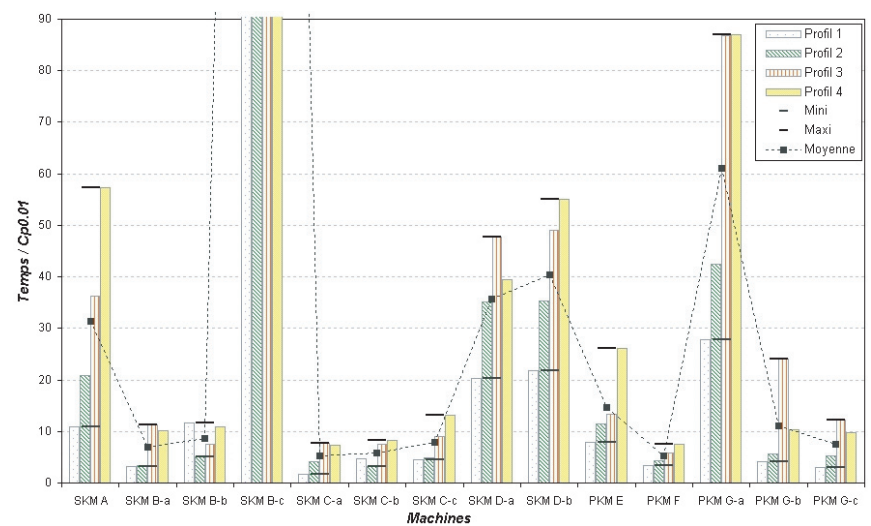

Fig. 9. Zoom sur le rapport Temps d'usinage $/ C p_{0,01}$.

De la même manière l'utilisation d'une tolérance CN de 10 micromètres sur la machine $S K M C$-b permet de réduire l'erreur de contour pour un temps d'usinage globalement identique à celui obtenu sur la machine $S K M C$-c (tolérance $\mathrm{CN}$ de 20 micromètres). Il en résulte un comportement de la machine plus homogène, en terme de productivité, sur les différents profils (Fig. 9) dans le cas $S K M$ $C$-b.

Enfin, les essais réalisés sur $S K M D$ permettent de conclure que l'activation du compactage des blocs $\mathrm{CN}$ (SKM D-a) permet de réduire le temps d'usinage au détriment de la précision, cependant d'un point de vue productivité (Fig. 9) la configuration $D-a$ reste la plus intéressante par rapport à la configuration $D-b$.

\subsection{Influence de la structure}

Nous avons montré dans le paragraphe précédent que les paramètres $\mathrm{FAO}$ et $\mathrm{CN}$ ont une influence non négligeable sur le couple temps/précision et qu'ils peuvent être optimisés afin d'utiliser au mieux la machine.

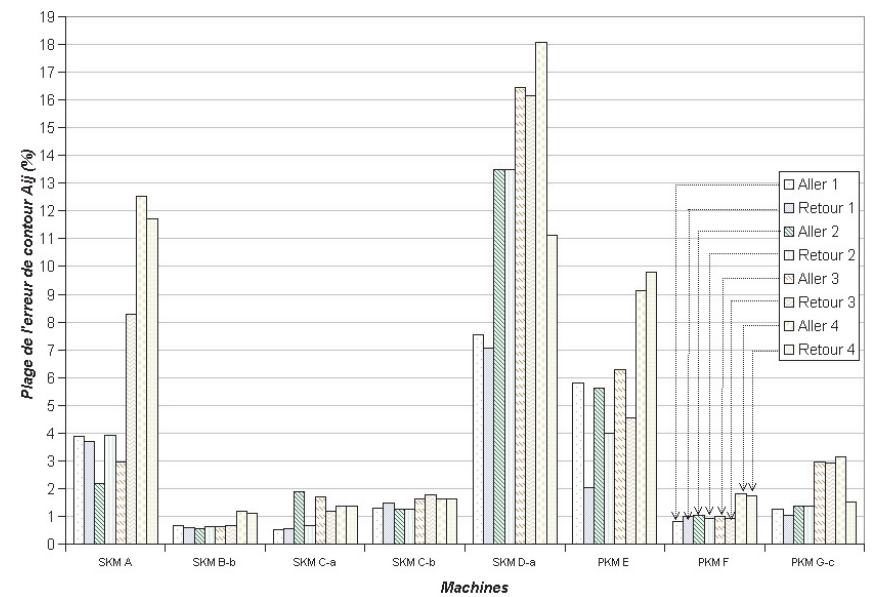

Fig. 10. Plage de l'erreur de contour en fonction du sens de parcours.

Les figures de cette section reprennent uniquement les meilleurs résultats pour chaque machine lorsque le critère retenu est celui de la productivité (exemple : $S K M D-a>S K M D-b)$. Ceci permet d'isoler et d'analyser l'influence de la structure. La figure 10 donne la précision et la figure 11 donne la productivité de ces huit machines, l'influence du sens de parcours (aller ou retour) étant également analysée.

Pour certaines structures de machines ( $S K M A$ et $P K M E$ ), le sens de parcours a des répercutions sur la précision. Ces essais peuvent donc permettre de choisir la stratégie d'usinage en fonction du critère retenu (temps, précision...). De plus, ces deux machines présentent également un comportement non homogène, puisque d'un profil à l'autre les erreurs de contour varient fortement.

Dans le cas où le critère retenu serait la productivité (Fig. 11), il apparaît clairement que les trois machines les plus performantes sont $S K M C-a, S K M C$-b et PKM F, avec - pour cette dernière - un comportement plus 


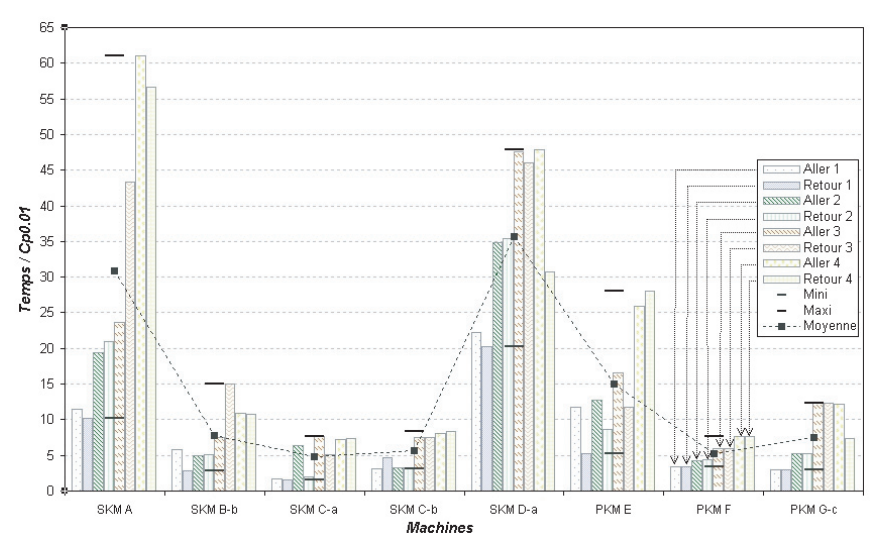

Fig. 11. Rapport Temps d'usinage $/ C p_{0,01}$ en fonction du sens de parcours.

homogène d'un profil à l'autre. Ce critère d'homogénéité est très important dans l'industrie où les pièces à produire peuvent être une combinaison des différentes formes présentent sur la pièce test. Par ailleurs, $S K M B$ - $b$ et $P K M G-c$ fournissent un niveau de productivité identique, avec une fois encore un comportement plus homogène pour la machine parallèle. PKM E a quant à elle un comportement relativement non homogène d'un profil et d'un sens de parcours à l'autre, tout comme $S K M A$ et $S K M D-a$. Ces trois dernières machines s'avèrent peu adaptées pour l'usinage de forme.

\section{Conclusion}

Depuis 1994, date de la présentation publique de la première machine-outil parallèle, très peu d'études ont été menées afin de tester leur aptitude en usinage et en particulier pour la réalisation de formes complexes.

Grâce à la méthode de mesure qui fait appel aux données codeurs, plusieurs machines parallèles ont pu être testées. Il a été montré dans un premier temps, que les paramètres $\mathrm{CN}$ et $\mathrm{FAO}$ ont une influence non négligeable sur la «qualité » de la pièce finale. Il est donc primordial de définir des procédures génériques afin de qualifier le comportement des machines. Le simulateur qui a été développé dans une étude précédente est un outil possible qui est en cours de développement pour les machines parallèles. Dans un second temps, nous avons pu montrer que les structures parallèles ont du potentiel pour l'usinage de formes complexes et pour l'UGV, puisque certaines d'entre-elles se sont avérées avoir des niveaux de précision et de productivité identique à certaines machines sérielles. Il est important de rappeler ici que l'objet de l'étude n'est pas de classer les machines les unes par rapport aux autres, mais de montrer que notre méthode permet de donner des indicateurs en terme de capabilité de machines, qu'elles soient sérielles ou parallèles. Par ailleurs, les tests et analyses tirées ne sont que le reflet des machines à l'instant où nous avons effectué les mesures.
L'étape suivante de cette étude, consiste à augmenter les conditions de coupe afin d'utiliser au mieux les potentialités dynamiques des structures parallèles. Ces nouveaux essais nous permettront de conclure quant à la productivité de telles machines pour voir si elles pourront un jour remplacer, ou au moins cohabiter avec les machines sérielles dans l'industrie.

\section{Références}

[1] A. Dugas, M. Terrier, J.Y. Hascoët, Free Form Surface Measurement Method and Machine Qualification for High Speed Milling, Int. Conf. on Integrated Design and Manufacturing in Mechanical Engineering, ClermontFerrand, France, 14-16 May, 2002, CD Rom

[2] J.P. Merlet, Les robots parallèles, Édition Hermès, 1990

[3] J.P. Merlet, An initiative for the kinematics study of parallel manipulators, Workshop on Fundamental Issues and Future Research Directions for Parallel Mechanisms and Manipulators, Quebec City, Canada, 3, 4 October, 2002, pp. 2-9

[4] I. Bonev, Delta Parallel Robot - the story of success, ParalleMIC, 2001, http://www.parallemic.org/ Reviews/Review002.html (Accès décembre 2003)

[5] F. Xi, D. Zhang, Z. Xu, C.M. Mechefske, A comparative study on tripod units for machine tools, Int. J. Machine Tools and Manufacture 43 (2003), 721-730

[6] S.A. Joshi, L.W. Tsai, The kinematics of a class of 3-DOF 4-Legged parallel manipulators, ASME 2002 Design Engineering Technical Conferences and Computer and Information in Engineering Conference, Montreal, Canada, 29 September-2 October, 2002, pp. 1-10

[7] A. Herrero, M. Zatarain, X. Almandoz, Development of a five-drive parallel kinematics machine, II. International Seminar on Improving Machine Tool Performance, La Baule, France, 3-5 July, 2000, CD Rom (ref. B16)

[8] http://www.smttricept.com (Accès décembre 2003)

[9] M. Weck, D. Staimer, Parallel Kinematic Machine Tools Current State and Future Potentials, Annals of the CIRP, $51 / 2(2002) 1-13$

[10] M. Geldart, P. Webb, H. Larsson, M. Backstrom, N. Gindy, K. Rask, A direct comparison of the machining performance of a variax 5 axis parallel kinetic machining centre with conventional 3 and 5 axis machine tools, Int. J. Machine Tools and Manufacture, in press

[11] A. Dugas, CFAO et UGV. Simulation d'usinage de formes complexes, Thèse de Doctorat, École centrale de Nantes, Université de Nantes, France, 13 décembre 2002

[12] J.Y. Hascoet, A. Dugas, J.J. Lee, Rapport Final Tâche III - Simulation des opérations d'usinage, Rapport final du projet «Saut technologique UGV », Institut de Recherche en Communications et Cybernétique de Nantes (IRCCyN), France, juin 2001, 161 p.

[13] Laboratoire Universitaire de Recherche en Production Automatisée, École normale supérieure de Cachan, 61 avenue du Président Wilson, 94235 Cachan Cedex, France, tél : +(33)1474022 15, fax : +(33)1474022 20

[14] E. Duc, C. Lartigue, S. Laporte, Assessment of the description format of tool trajectories in 3-axis HSM, Third International Conference on Metal cutting and High Speed Machining, Metz, France, June 2001, pp. CANC6 